\title{
Diagnostic Value of Serum Markers Combined with TVCDS in Ovarian Cancer Patients Treated with Bushen Yiqi Quyu Prescription
}

\author{
Wei Qin $\mathbb{D}^{1},{ }^{1}$ Fengmin Jiang $\mathbb{D}^{2},{ }^{2}$ and Tao Zhang $\mathbb{C}^{3}$ \\ ${ }^{1}$ Department of Ultrasound Medicine, Zaozhuang Mental Health Center, Zaozhuang 277103, China \\ ${ }^{2}$ Department of Ultrasound Medicine, Maternity and Child Health Care of Zaozhuang, Zaozhaung 277100, China \\ ${ }^{3}$ Department of Ultrasound Medicine, Tengzhou Central People's Hospital, Tengzhou 277599, China \\ Correspondence should be addressed to Tao Zhang; zhangtaotengzhou@163.com
}

Received 27 September 2021; Accepted 29 October 2021; Published 13 November 2021

Academic Editor: Muhammad Wasim Khan

Copyright $(2021$ Wei Qin et al. This is an open access article distributed under the Creative Commons Attribution License, which permits unrestricted use, distribution, and reproduction in any medium, provided the original work is properly cited.

Background. To compare the diagnostic value of serum markers human epididymal protein 4 (HE4) and cyclooxygenase-1 (COX1) combined with transvaginal color Doppler sonography (TVCDS) in ovarian cancer (OC) treated with Bushen Yiqi Quyu prescription. Methods. A total of 232 OC patients treated at the hospital from January 2018 to October 2020 were randomly divided into an observation group $(n=116)$ and control group $(n=116)$. The control group was treated with essential Western medication, and the observation group was treated with essential Western medication and Bushen Yiqi Quyu prescription. The clinical efficacy of the two groups was compared. The levels of HE4 and COX-1 were compared between the two groups before and after treatment. The ultrasonic features of TVCDS were compared between the two groups before and after treatment. The ROC curve was drawn to compare the sensitivity, specificity, and accuracy of single and combined detection of HE4, COX-1, and TVCDS in the observation group. Results. The total effective rate of the observation group was significantly higher than that of the control group. After treatment, HE4 and COX-1 levels in both groups were considerably lower than those before treatment, and in the observation group, they decreased significantly than in the control group. HE4 and COX-1 were positively correlated with the clinical stage of OC. The higher the clinical stage, the higher the levels of HE4 and COX-1. After treatment, there was no significant difference in tumor location and the boundary between the two groups. There were statistically significant differences in tumor echo, nature, morphology, calcification, internal blood flow, and lymph node metastasis, and the difference in the observation group was more evident than in control group. The sensitivity, specificity, accuracy, positive detection rate, and negative detection rate of combined detection were higher than those of single detection. Conclusions. Bushen Yiqi Quyu prescription has certain curative effects in the treatment of OC patients, which can significantly reduce the level of tumor markers and improve the symptoms of OC patients. The combined detection of HE4, COX-1, and TVCDS has high sensitivity, specificity, and accuracy, which can effectively detect $\mathrm{OC}$ and reduce missed diagnosis and misdiagnosis.

\section{Introduction}

Ovarian cancer $(\mathrm{OC})$ is one of the common malignant tumors of the female reproductive system, and its incidence varies in the world. In China, the incidence of OC is high and on the rise, second only to endometrial cancer and cervical cancer [1]. Clinically, epithelial ovarian cancer has a high mortality rate, ranking first among female reproductive system malignant tumors [2]. The incidence of OC is insidious, and most clinically diagnosed patients are in the middle and late stages. The 5-year survival rate is only about $30 \%$, posing a serious threat to patients' life [3]. Therefore, early screening can timely detect the disease and provide an effective treatment plan to patients. It has been reported that if patients can be diagnosed at stage I-II, the 5-year survival rate increases from $30 \%$ to more than $70 \%$ [4]. Therefore, finding an accurate and effective diagnostic method is the key to early diagnosis and treatment of OC. 
HE4 is a new marker for OC. Studies have found that HE4 is highly expressed in OC cells, predominantly serous and ovarian endometrioid tumors, and currently plays an essential role in the early diagnosis, prognosis, and disease monitoring of OC [5]. COX-1 is a new ovarian tumor-associated antigen, which exists in some OC and cervical cancer cell lines. COX-1 is not expressed in healthy tissues, but is closely related to OC tissues [6]. At present, transvaginal color Doppler sonography (TVCDS) is a commonly used imaging examination method in diagnosing OC. It has the advantages of simple operation, being economical and noninvasive, and displaying the blood supply characteristics of tumors through CDFI, which is widely used in the screening and diagnosis of gynecological diseases [7].

The treatment of OC is mainly surgery, supplemented by radiotherapy and chemotherapy. However, long-term use of chemotherapy drugs can damage normal cells and cause a series of adverse reactions, seriously affecting the normal life of patients [8]. Studies in recent years have shown [9] that TCM has achieved remarkable results in adjuvant chemotherapy for tumor treatment, which can not only improve the efficacy but also alleviate the adverse reactions caused by chemotherapy and enhance the immune function of the body. In TCM, OC could be classified as "accumulation," "abdominal mass," and "female abdominal mass" [10]. The occurrence of OC is caused by the weakness of the body resulting in low vitality [11]. Wu et al. [12] claimed that the pathogenesis of OC was due to the deficiency of vital qi and poor blood flow, and treatment should focus on replenishing qi and removing blood stasis, with Shi Quan Da Bu decoction as the prescription. Wang Y et al. [13] reported that the etiology and pathogenesis of OC were the imbalance of Yin and Yang in the body, the obstruction of blood flow, and the accumulation of blood stasis. The treatment should be combined with the combination of strengthening and removing pathogenic factors. Bushen Yiqi Quyu prescription can not only reduce the adverse reactions of radiotherapy and chemotherapy but also enhance the immune function of the body, to improve the therapeutic effects against tumor.

The purpose of this study was to observe the diagnostic value of HE4 and COX-1 combined with TVCDS in OC patients treated with Bushen Yiqi Quyu prescription.

\section{Materials and Methods}

2.1. General Information. A total of $232 \mathrm{OC}$ patients treated at the Tengzhou Central People's Hospital, Tengzhou, Shandong, China, from January 2018 to October 2020 were randomly divided into an observation group $(n=116)$ and control group $(n=116)$. Inclusion criteria: (1) diagnosed by histopathology; (2) met the Western medicine diagnostic criteria [14] and TCM syndrome differentiation criteria [15]; (3) have not undergone other gynecological operations; and (4) the expected survival time is more than 6 months. Exclusion criteria: (1) patients allergic to medication used in this study; (2) complicated with other gynecological diseases and benign and malignant tumors; (3) have mental or communication disorders, unable to communicate; and (4) incomplete case data. The informed consent of all patients was obtained and approved by the ethics committee of the Tengzhou Central People's Hospital, Tengzhou, Shandong, China. There was no significant difference in general data between the two groups (Table 1).

2.2. The Treatment. The control group was treated with prescribed Western medicine (including surgery and radiotherapy and chemotherapy in routine), and the observation group was given Bushen Yiqi Quyu prescription on the basis of the treatment in the control group. The ingredients of Bushen Yiqi Quyu prescription are $30 \mathrm{~g} \mathrm{Scu-}$ tellariae Barbatae Herba, $30 \mathrm{~g}$ Fallopia multiflora, $30 \mathrm{~g}$ Dioscoreae Rhizoma, 30 Rehmanniae Radix Praeparata, $20 \mathrm{~g}$ Scutellaria baicalensis, 20 g Codonopsis Radix, 15 g Ligustri Lucidi Fructus, $15 \mathrm{~g}$ Chuanxiong Rhizoma, $15 \mathrm{~g}$ Corni Fructus, $15 \mathrm{~g}$ Solanum nigrum, $15 \mathrm{~g}$ Curcumae Rhizoma, $15 \mathrm{~g}$ Poria, $15 \mathrm{~g}$ Herba Hedyotidis, and $6 \mathrm{~g}$ Glycyrrhizae Radix et Rhizoma Praeparata cum Melle. This prescription was decocted with water and taken warm on an empty stomach in the morning and evening, $200 \mathrm{~mL} /$ time, once per $1 \mathrm{~d}$. The treatment period was $21 \mathrm{~d}$, and the treatment period was 3 .

2.3. Observed Indicators. Clinical efficacy [16]: complete remission (CR), all lesions disappeared, and no new lesions were observed for more than 1 month; partial remission (PR), the lesions were $1 / 2$ smaller than before chemotherapy, and there was no lesion enlargement; stable disease (SD), the lesion was without shrinkage or enlargement; and progression disease (PD), the lesions increased by $1 / 4$ compared with before treatment, or new lesions were seen. Total effective rate was calculated using the following equation:

$$
\text { Total effective rate }=\left[\frac{(\mathrm{CR}+\mathrm{PR})}{\text { total number of cases }}\right] \times 100 \% \text {. }
$$

2.4. Determination of Serum Marker Levels. $5 \mathrm{ml}$ fasting venous blood was collected from the two groups in the morning and made to stand at room temperature for $30 \mathrm{~min}$. The serum was separated by centrifugation and stored in a refrigerator at $-20^{\circ} \mathrm{C}$. $\mathrm{HE} 4$ level was detected by enzymelinked immunosorbent assay (ELISA) (Kananga, Sweden). The cox-1 level was measured by electrochemiluminescence (Roche). All were operated in strict accordance with the manual requirements, and all quality control met the criteria. The criteria were COX-1 $>12 \mathrm{U} / \mathrm{mL}$ was positive and $\mathrm{HE} 4>150 \mathrm{pmol} / \mathrm{L}$ was positive.

2.5. TVCDS. The PHILIPS iU22 color Doppler ultrasound diagnostic instrument with a probe frequency of $7.5 \mathrm{MHz}$ was used for TVCDS. The patient emptied the bladder and probed the uterus and adnexa area with an intracavitary probe through the vagina. The size and internal structure of the adnexa tumor were carefully scanned to observe the size, location, boundary, shape, and internal echo of the tumors. 
TABle 1: Comparison of general clinical data between the two groups.

\begin{tabular}{|c|c|c|c|c|}
\hline & Observation group $(n=116)$ & Control group $(n=116)$ & $X^{2}$ & $p$ \\
\hline Age (years) & & & 0.075 & 0.784 \\
\hline$>50$ & 74 & 76 & & \\
\hline$\leq 50$ & 42 & 40 & & \\
\hline Weight (kg) & & & 0.163 & 0.687 \\
\hline$>62$ & 69 & 72 & & \\
\hline$\leq 62$ & 47 & 44 & & \\
\hline The course of OC (years) & & & 0.309 & 0.578 \\
\hline$>2$ & 37 & 41 & & \\
\hline$\leq 2$ & 79 & 75 & & \\
\hline Symptom & & & 0.086 & 0.769 \\
\hline Obvious abdominal pain & 83 & 85 & & \\
\hline No obvious symptoms & 33 & 31 & & \\
\hline Clinical stage & & & 0.177 & 0.981 \\
\hline I & 27 & 29 & & \\
\hline II & 35 & 34 & & \\
\hline III & 43 & 41 & & \\
\hline IV & 11 & 12 & & \\
\hline Pathological type & & & 0.395 & 0.941 \\
\hline Serous cystadenocarcinoma & 78 & 75 & & \\
\hline Endometrial carcinoma & 7 & 6 & & \\
\hline Mucinous cystadenocarcinoma & 19 & 22 & & \\
\hline Others & 12 & 13 & & \\
\hline
\end{tabular}

2.6. Statistical Analysis. SPSS 22.0 software was used for statistical analysis. Measurement data were expressed by mean \pm standard deviation, and comparison between groups was performed by the $t$-test. Count data were expressed by $[N(\%)]$, and comparison between groups was performed by the $\chi^{2}$ test. An ROC curve was drawn to calculate sensitivity and specificity. $p<0.05$ was considered statistically significant.

\section{Results}

3.1. Comparison of Clinical Efficacy between the Two Groups. The total effective rate of the observation group was $83.62 \%$, significantly higher than that of the control group $62.93 \%$ (Table 2). The results show that Bushen Yiqi Quyu prescription can improve the curative effects.

\subsubsection{Comparison of Serum Tumor Marker Levels between the} Two Groups before and after Treatment. Before treatment, there was no significant difference in serum HE4 and COX-1 levels between two groups. After treatment, HE4 and COX-1 of the observation group were $(106.01 \pm 46.58) \mathrm{pmol} / \mathrm{L}$ and $(11.05 \pm 3.11) \mathrm{U} / \mathrm{mL}$, respectively. The levels of $\mathrm{HE} 4$ and COX-1 in the control group were $(156.32 \pm 47.17) \mathrm{pmol} / \mathrm{mL}$ and $(26.03 \pm 4.68) \mathrm{U} / \mathrm{mL}$, respectively. After treatment, the levels of HE4 and COX-1 were significantly lower than before treatment. After treatment, the levels of HE4 and COX-1 in the observation group were lower than those in the control group (Figure 1).

3.1.2. Comparison of Serum Tumor Markers in Patients with Different Stages of OC. The results showed that the levels of HE4 and COX-1 were positively correlated with the clinical stage of OC. The higher the clinical stage, the higher the levels of HE4 and COX-1 (Table 3).

3.1.3. Comparison of the Ultrasonic Features in OC Patients before and after Treatment between the Two Groups. Before treatment, there was no significant difference in ultrasound indexes between the two groups. After treatment, there were statistically significant differences in echo, nature, morphology, calcification, internal blood flow, and lymph node metastasis between the two groups, and the difference in the observation group was more obvious than in the control group (Table 4).

3.1.4. Comparison of the Diagnostic Value of HE4, COX-1, and TVCDS in Single and Combined Detection of OC. The sensitivity, specificity, accuracy, positive rate, and negative rate of COX-1 in the diagnosis of OC were $65.38 \%, 81.58 \%$, $70.69 \%, 87.93 \%$, and $55.17 \%$, respectively. The sensitivity, specificity, accuracy, positive rate, and negative rate of HE4 in the diagnosis of OC were $73.08 \%, 84.21 \%, 76.72 \%, 90.48 \%$, and $60.38 \%$, respectively. The sensitivity, specificity, accuracy, positive rate, and negative rate of TVCDS in the diagnosis of OC were $67.95 \%, 86.84 \%, 74.14 \%, 91.38 \%$, and $59.90 \%$, respectively. The sensitivity, specificity, accuracy, positive rate, and negative rate of combined detection were $92.31 \%, 92.11 \%$, $92.24 \%, 96.00 \%$, and $85.37 \%$, respectively. The results showed that the combined detection was higher than the single detection, and the difference in sensitivity, accuracy, and negative detection rate was statistically significant (Figure 2 and Table 5).

\section{Discussion}

OC is one of the common malignant tumors of the female reproductive system and has the highest mortality rate 
TABLE 2: Comparison of clinical efficacy between the two groups.

\begin{tabular}{lcccccc}
\hline Group & $n$ & CR & PR & SD & PD & Total effective rate \\
\hline Observation group & 116 & $38(32.76)$ & $59(50.86)$ & $13(11.21)$ & $6(5.17)$ & $97(83.62)$ \\
Control group & 116 & $21(18.10)$ & $52(44.83)$ & $27(23.28)$ & $16(13.79)$ & $73(62.93)$ \\
$X^{2}$ & & & & & 14.785 & 0.002 \\
\multicolumn{1}{r}{$p$} & & & & & & \\
\hline
\end{tabular}

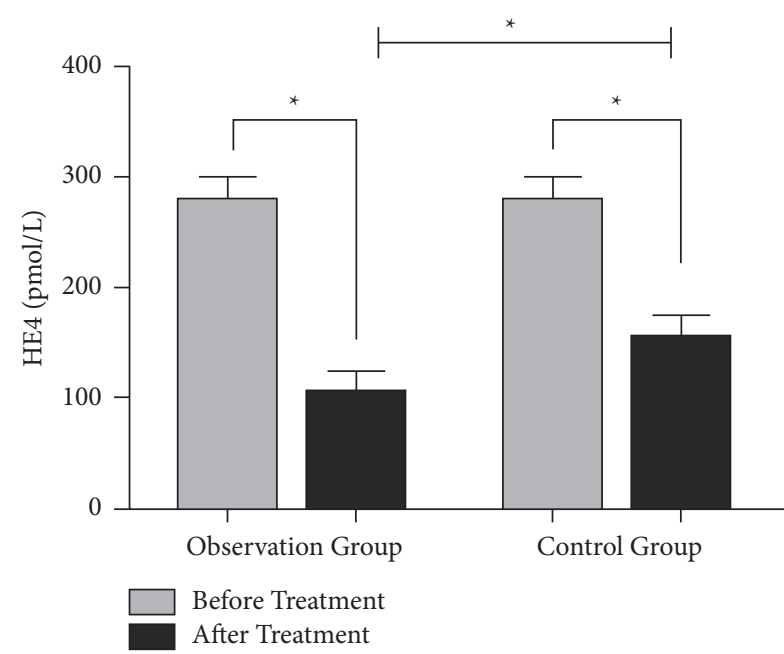

(a)

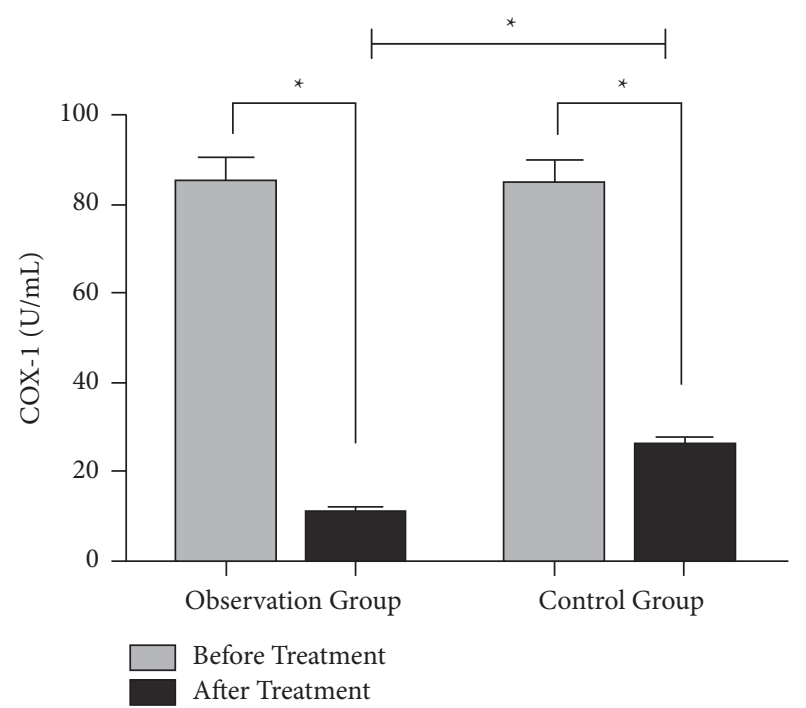

(b)

FIGURE 1: Comparison of serum tumor marker levels between the two groups before and after treatment. (a) comparison of HE4 between the two groups before and after treatment. (b) The comparison of COX-1 between the two groups before and after treatment. ${ }^{*} p<0.05$.

TABLE 3: Comparison of serum tumor markers in patients with different stages of OC.

\begin{tabular}{lccc}
\hline Clinical stage & $n$ & HE4 $(\mathrm{pmol} / \mathrm{L})$ & COX-1 $(\mathrm{U} / \mathrm{mL})$ \\
\hline I & 56 & $193.52 \pm 41.64$ & $46.32 \pm 8.65$ \\
II & 69 & $227.83 \pm 43.12$ & $65.94 \pm 9.28$ \\
III & 84 & $278.96 \pm 38.57$ & $91.76 \pm 11.37$ \\
IV & 23 & $353.67 \pm 32.86$ & $136.54 \pm 13.71$ \\
$t$ & & 8.623 & 7.540 \\
$p$ & & $<0.05$ & $<0.05$ \\
\hline
\end{tabular}

among gynecological tumors. As the early clinical symptoms are not apparent, it is easy to cause missed diagnosis and misdiagnosis, so most OC patients are found with the advanced stage at the time of diagnosis commonly [17]. OC is difficult to treat, with surgery and chemotherapy as the main treatment, but the prognosis is poor and the recurrence rate is high [18]. Chemotherapy has therapeutic effects, but it usually causes severe side effects. According to Chinese traditional medicine, the kidney is the innate book and the spleen is the root of acquired. The spleen and kidney can be treated together to nourish the viscera and restore vital qi [19]. If vital qi is deficient, kidney qi will become further weak and the body will produce Yin and Yang imbalance to aggravate the disease. Health professionals have different opinions about treating OC. Francisco Fernandez et al. [20] believed that invigorating the spleen and tonifying the kidney could improve the fatigue symptoms of OC patients. Tao et al. reported [21] that the treatment of OC should be based on disease differentiation, constitution differentiation, and combination of disease differentiation and syndrome differentiation.

According to our findings, the total effective rate in the observation group was significantly higher than that in the control group. HE4 and COX-1 levels in the observation group were predominantly higher than those in the control group after therapy. After treatment, there were significant differences between the two groups in tumor echo, nature, morphology, calcification, internal blood flow, and lymph node metastasis. This is because Rehmanniae Radix Praeparata, Corni Fructus, and Dioscoreae Rhizoma have a nourishing role. Corni Fructus can tonify the liver and kidney and cure frequent urination, backache, 
TABLE 4: Comparison of ultrasonographic features of TVCDS in OC patients before and after treatment between the two groups.

\begin{tabular}{|c|c|c|c|c|c|c|c|}
\hline \multirow{2}{*}{\multicolumn{2}{|c|}{ Ultrasonographic features }} & \multicolumn{2}{|c|}{ Observation group } & \multicolumn{2}{|c|}{ Control group } & \multirow[b]{2}{*}{$X^{2}$} & \multirow[b]{2}{*}{$p$} \\
\hline & ic features & $\begin{array}{l}\text { Before treatment } \\
\qquad(n=116)\end{array}$ & $\begin{array}{l}\text { After treatment } \\
\quad(n=78)\end{array}$ & $\begin{array}{l}\text { Before treatment } \\
\quad(n=116)\end{array}$ & $\begin{array}{l}\text { After treatment } \\
\quad(n=95)\end{array}$ & & \\
\hline \multirow[t]{2}{*}{ Location } & $\begin{array}{l}\text { Above the } \\
\text { middle }\end{array}$ & 74 & 52 & 69 & 57 & \multirow[t]{3}{*}{1.347} & \multirow[t]{3}{*}{$>0.05$} \\
\hline & Below & 42 & 26 & 47 & 38 & & \\
\hline \multirow[b]{2}{*}{ Echo } & Hypoechoic & 87 & 31 & 85 & 52 & & \\
\hline & $\begin{array}{l}\text { High or equal } \\
\text { echo }\end{array}$ & 29 & 47 & 31 & 43 & 33.288 & $<0.05$ \\
\hline \multirow{2}{*}{ Boundary } & Clarity & 79 & 55 & 82 & 74 & \multirow{2}{*}{2.642} & \multirow{2}{*}{$>0.05$} \\
\hline & Obscure & 37 & 23 & 34 & 21 & & \\
\hline \multirow{3}{*}{ Characters } & Solid & 47 & 16 & 44 & 27 & \multirow{3}{*}{40.247} & \\
\hline & Cyst-solid & 42 & 13 & 43 & 29 & & \multirow[t]{2}{*}{$<0.05$} \\
\hline & Cystic & 27 & 49 & 29 & 39 & & \\
\hline \multirow{2}{*}{ Shape } & Round or oval & 49 & 23 & 53 & 37 & \multirow{2}{*}{5.443} & \multirow{2}{*}{$<0.05$} \\
\hline & Irregular & 67 & 55 & 63 & 58 & & \\
\hline \multirow{3}{*}{ Calcification } & No & 12 & 25 & 15 & 23 & \multirow{3}{*}{54.532} & \\
\hline & Tiny & 38 & 43 & 40 & 46 & & \multirow{2}{*}{$<0.05$} \\
\hline & Bulky & 66 & 10 & 61 & 26 & & \\
\hline \multirow{2}{*}{ Internal blood flow } & Not rich & 41 & 53 & 44 & 51 & \multirow{2}{*}{25.605} & \multirow{2}{*}{$<0.05$} \\
\hline & Rich & 75 & 25 & 72 & 44 & & \\
\hline \multirow{2}{*}{$\begin{array}{l}\text { Lymph node } \\
\text { metastasis }\end{array}$} & No & 51 & 66 & 53 & 60 & \multirow{2}{*}{39.780} & \multirow{2}{*}{$<0.05$} \\
\hline & Yes & 65 & 12 & 63 & 35 & & \\
\hline
\end{tabular}

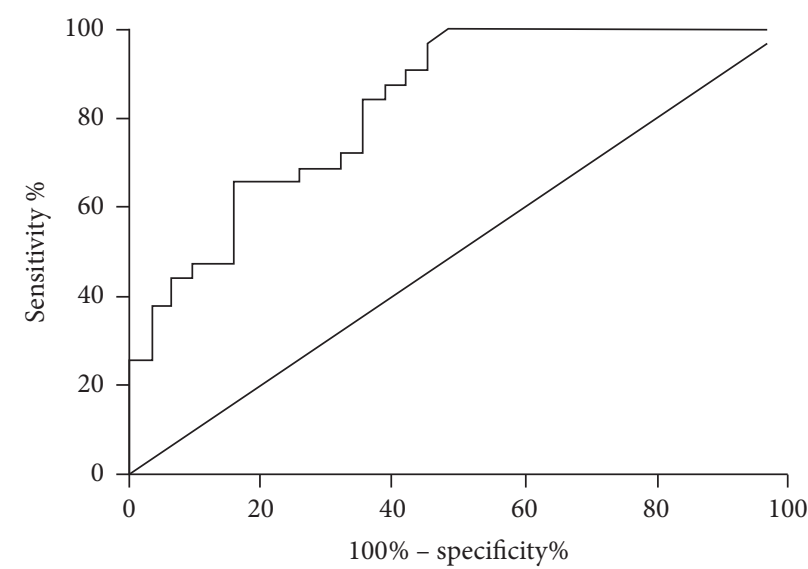

(a)

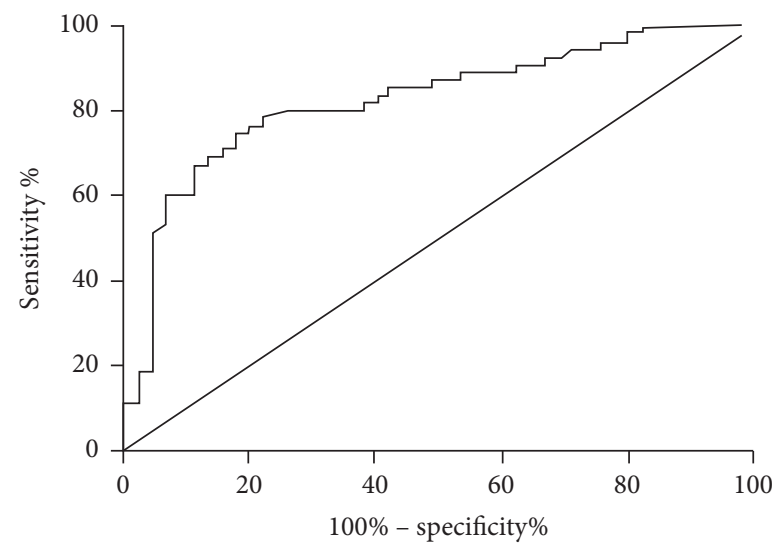

(c)

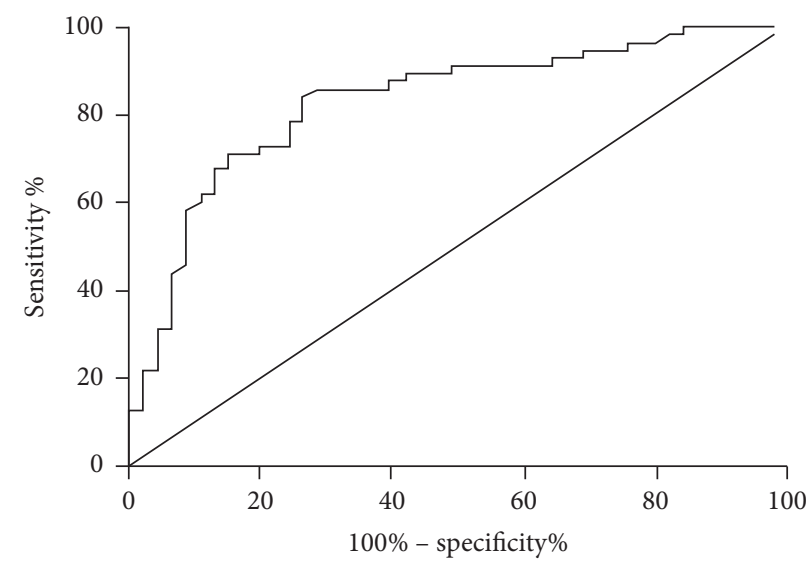

(b)

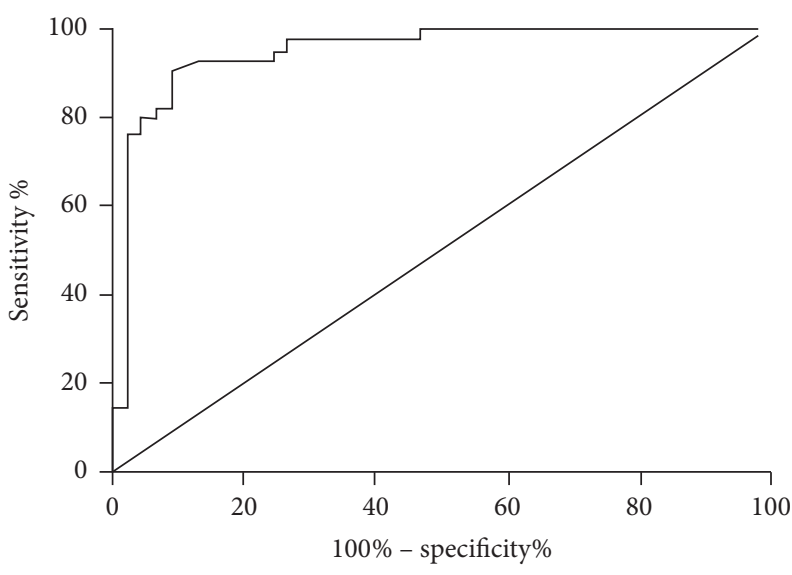

(d)

Figure 2: ROC curves of HE4, COX-1, and TVCDS for single and combined detection of OC. (A) ROC curve of COX-1 for the diagnosis of OC. (B) ROC curve of HE4 in the diagnosis of OC. (C) ROC curve of TVCDS in the diagnosis of OC. (D) ROC curve of the combined diagnosis of OC. 
TABle 5: Diagnostic value of HE4, COX-1, and TVCDS in the single and combined detection of OC.

\begin{tabular}{|c|c|c|c|c|c|}
\hline & Sensitivity & Specificity & Accuracy & Positive rate & Negative rate \\
\hline HE4 & $73.08(57 / 78)$ & $84.21(32 / 38)$ & $76.72(89 / 116)$ & $90.48(57 / 63)$ & $60.38(32 / 53)$ \\
\hline COX-1 & $65.38(51 / 78)$ & $81.58(31 / 38)$ & $70.69(82 / 116)$ & $87.93(51 / 58)$ & $55.17(32 / 58)$ \\
\hline TVCDS & $67.95(53 / 78)$ & $86.84(33 / 38)$ & $74.14(86 / 116)$ & $91.38(53 / 58)$ & $56.90(33 / 58)$ \\
\hline Combined detection & $92.31(72 / 78)$ & $92.11(35 / 38)$ & $92.24(107 / 116)$ & $96.00(72 / 75)$ & $85.37(35 / 41)$ \\
\hline
\end{tabular}

dysmenorrhea, and vaginal bleeding. Dioscoreae Rhizoma can nourish the spleen and strengthen the kidney. Rehmanniae Radix Praeparata can nourish Yin and blood. Ligustri Lucidi Fructus can nourish kidney Yin. Codonopsis Radix can tonify qi and enhance immunity. Chuanxiong Rhizoma can promote qi, activate blood, and dispel wind for dispelling pain. Solanum nigrum can remove heat and eliminate toxicity and dissipate blood stasis for detumescence. Curcumae Rhizoma can remove blood stasis and relieve pain and protect the liver and kidney. Poria can nourish the heart to tranquilize, reinforce $\mathrm{Qi}$, and strengthen the spleen. Scutellariae Barbatae Herba can clear heat and remove toxicity and boost blood circulation for removing blood stasis and detumescence for relieving pain. Fallopia multiflora has the effect of reinforcing the kidney for supplementing essence and detoxification. Herba Hedyotidis and Scutellaria baicalensis can clear heat and remove toxicity and promote urination. Glycyrrhizae Radix et Rhizoma Praeparata cum Melle has the effect of benefiting qi for nourishing yin and activating Yang [22-24]. Choi et al. [25] found that Scutellaria baicalensis can effectively inhibit the proliferation and induce apoptosis of OC cells. Ruanet al. [26] found that Poria cocos extract can inhibit the occurrence and development of OC by interfering with mitochondrial function, galactose, and fatty acid metabolism. All these remedies can improve the clinical symptoms of OC patients and promote their recovery by boosting blood circulation and removing blood stasis, resolving static blood for relieving pain, diuresis, tonifying the kidney and spleen, tonifying qi, and moistening the lung.

Early screening of OC is of great significance. Serum markers play an important role in diagnostic oncology. In recent years, many potential markers have been identified and used individually or in combination to improve specificity and sensitivity, especially in the early stages of disease [27]. TVCDS is also commonly used in the diagnosis of gynecological carcinomas. It is characterized by the ability to precisely display the blood flow signals in the lesions and can directly observe the patient's ovarian morphology, size, and abnormal surrounding tissues, so as to accurately evaluate the patient's tumor. It is noninvasive, easy to operate, and economical and can be used as an important auxiliary approach for the clinical examination of OC $[28,29]$. The results showed that serum HE4 and COX-1 levels increased gradually with the increase of clinical stage, indicating that the two serum markers can assist the early diagnosis of OC. In this study, the sensitivity, specificity, and accuracy of HE4 and COX-1 combined with TVCDS were significantly higher than those of either assay alone. Combined detection can further improve the diagnosis rate and reduce the rate of missed diagnosis and misdiagnosis. However, the sample size of this study is small, and the results may be biased to some extent. It is necessary to increase the sample size for further research in the future.

\section{Conclusions}

In conclusion, Bushen Yiqi Quyu prescription in this study has good therapeutic effects in treating OC, which can significantly reduce the serum indexes of patients and improve the tumor deterioration. The application of TVCDS combined with HE4 and COX-1 in the treatment of OC is optimum and significantly higher than each single detection, which has potential of clinical application for the early diagnosis of OC.

\section{Data Availability}

The data used to support the findings of this study are available from the corresponding author upon request.

\section{Conflicts of Interest}

The authors declare no conflicts of interest.

\section{References}

[1] K. Odunsi, "Immunotherapy in ovarian cancer," Annals of Oncology: Official Journal of the European Society for Medical Oncology, vol. 28, no. S8, 2017.

[2] S. Lheureux, M. Braunstein, and A. M. Oza, "Epithelial ovarian cancer: evolution of management in the era of precision medicine," CA: A Cancer Journal for Clinicians, vol. 69, no. 4, pp. 280-304, 2019.

[3] K. Aleksandra Kujawa and K. M. Lisowska, "Ovarian cancer-from biology to clinic," Postępy Higieny i Medycyny Doświadczalnej, vol. 69, pp. 1275-1290, 2015.

[4] K. M. Elias, J. Guo, and R. C. Bast Jr., "Early detection of ovarian cancer," Hematology-Oncology Clinics of North America, vol. 32, no. 6, pp. 903-914, 2018.

[5] V. Dochez, H. Caillon, E. Vaucel, J. Dimet, N. Winer, and G. Ducarme, "Biomarkers and algorithms for diagnosis of ovarian cancer: CA125, HE4, RMI and ROMA, a review," Journal of Ovarian Research, vol. 12, no. 1, p. 28, 2019.

[6] A. J. Wilson, O. Fadare, A. Beeghly-Fadiel et al., "Aberrant over-expression of COX-1 intersects multiple pro-tumorigenic pathways in high-grade serous ovarian cancer," Oncotarget, vol. 6, no. 25, pp. 21353-21368, 2015.

[7] J. T. Henderson, E. M. Webber, and G. F. Sawaya, "Screening for ovarian cancer," Journal of the American Medical Association, vol. 319, no. 6, pp. 595-606, 2018.

[8] E. A. Eisenhauer, "Real-world evidence in the treatment of ovarian cancer," Annals of Oncology: Official Journal of the European Society for Medical Oncology, vol. 28, no. S8, 2017. 
[9] D. W. Chan, M. M. Yung, Y.-S. Chan et al., "MAP30 protein from Momordica charantia is therapeutic and has synergic activity with cisplatin against ovarian cancer in vivo by altering metabolism and inducing ferroptosis," Pharmacological Research, vol. 161, Article ID 105157, 2020.

[10] Y. Liu, S. Gao, J. Zhu, Y. Zheng, H. Zhang, and H. Sun, "Dihydroartemisinin induces apoptosis and inhibits proliferation, migration, and invasion in epithelial ovarian cancer via inhibition of the hedgehog signaling pathway," Cancer Medicine, vol. 7, no. 11, pp. 5704-5715, 2018.

[11] J. Sun, J. Liu, D. Liu, and X. Wu, "Network pharmacologybased and clinically relevant prediction of the potential targets of Chinese herbs in ovarian cancer patients," BioMed Research International, vol. 2020, Article ID 8965459, 2020.

[12] Y. H. Wu, H. Y. Chen, C. H. Lai et al., "Use of Chinese herbal medicine improves chemotherapy-induced thrombocytopenia among gynecological cancer patients: an observational study," Evidence-based Complementary and Alternative Medicine: ECAM, vol. 2018, Article ID 4201325, 8 pages, 2018.

[13] Y. Wang, Y. Li, Y. Zhang et al., "Multi-dimensional spectrumeffect relationship of the impact of Chinese herbal formula Lichong shengsui yin on ovarian cancer," Molecules, vol. 22, no. 6 , p. 979, 2017.

[14] S. Hauptmann, K. Friedrich, R. Redline, and S. Avril, “Ovarian borderline tumors in the 2014 WHO classification: evolving concepts and diagnostic criteria," Virchows Archiv, vol. 470, no. 2, pp. 125-142, 2017.

[15] I. K. Tsui, W. C. Dodson, A. R. Kunselman et al., "Chinese Obstetrics \& Gynecology journal club: a randomised controlled trial," BMJ Open, vol. 6, no. 1, Article ID e010178, 2016.

[16] C. A. Doubeni, A. R. Doubeni, and A. E. Myers, "Diagnosis and management of ovarian cancer," American Family Physician, vol. 93, no. 11, pp. 937-944, 2016.

[17] M. Kossaï, A. Leary, J. Y. Scoazec, and C. Genestie, "Ovarian cancer: a heterogeneous disease," Pathobiology: Journal of Immunopathology, Molecular and Cellular Biology, vol. 85, no. 1-2, pp. 41-49, 2018.

[18] A. Súarez-Zaizar, E. Cárdenas-Cárdenas, Y. A. Barajas-Castro, and P. Cortés-Esteban, "Controversies on the treatment of ovarian cancer with dose-dense chemotherapy," Chinese Clinical Oncology, vol. 9, no. 4, p. 53, 2020.

[19] L. Han, X. Guo, H. Bian et al., "Guizhi fuling wan, a traditional Chinese herbal formula, sensitizes cisplatin-resistant human ovarian cancer cells through inactivation of the PI3K/AKT/ mTOR pathway," Evidence-based Complementary and Alternative Medicine: ECAM, vol. 2016, Article ID 4651949, 11 pages, 2016.

[20] M. Francisco Fernandez, C. Charfi, J. Piloto-Ferrer, M. Lidia González, S. Lamy, and B. Annabi, "Targeting ovarian cancer cell cytotoxic drug resistance phenotype with Xanthium strumarium L. extract," Evidence-Based Complementary and Alternative Medicine, vol. 2019, Article ID 6073019, 13 pages, 2019.

[21] F. Tao, S. Ruan, W. Liu, L. Wang, Y. Xiong, and M. Shen, "Fuling granule, a traditional Chinese medicine compound, suppresses cell proliferation and TGF $\beta$-induced EMT in ovarian cancer," PLoS One, vol. 11, no. 12, Article ID e0168892, 2016.

[22] S. Ge, Q. Xing, A. Zhang, and Y. Wang, "Effect of traditional Chinese medicine (TCM) on survival, quality of life, and immune function in patients with ovarian carcinoma," Medicine, vol. 100, no. 2, Article ID e23904, 2021.

[23] D. Liu, X. Meng, D. Wu, Z. Qiu, and H. Luo, "A natural isoquinoline alkaloid with antitumor activity: studies of the biological activities of berberine," Frontiers in Pharmacology, vol. 10, p. 9, 2019.

[24] K. K. L. Chan, T. J. Yao, B. Jones et al., "The use of Chinese herbal medicine to improve quality of life in women undergoing chemotherapy for ovarian cancer: a double-blind placebo-controlled randomized trial with immunological monitoring," Annals of Oncology, vol. 22, no. 10, pp. 2241-2249, 2011.

[25] B. Y. Choi, J. C. Joo, Y. K. Lee, I.-S. Jang, S. J. Park, and Y. J. Park, "Anti-cancer effect of Scutellaria baicalensis in combination with cisplatin in human ovarian cancer cell," BMC Complementary and Alternative Medicine, vol. 17, no. 1, p. $277,2017$.

[26] S. Ruan, Z. Zhang, X. Tian et al., "Compound fuling granule suppresses ovarian cancer development and progression by disrupting mitochondrial function, galactose and fatty acid metabolism," Journal of Cancer, vol. 9, no. 18, pp. 3382-3393, 2018.

[27] X. Dong, X. Men, W. Zhang, and P. Lei, "Advances in tumor markers of ovarian cancer for early diagnosis," Indian Journal of Cancer, vol. 51, no. S3, pp. e72-6, 2014.

[28] C. Yu, T. Dou, Y. Liu, and R. Liu, "Clinical value of TV-CDS combined with serum tumor markers in diagnosis of ovarian cancer," Oncology Letters, vol. 20, no. 2, pp. 2028-2034, 2020.

[29] A. C. Fleischer, A. Lyshchik, M. Hirari, R. D. Moore, R. G. Abramson, and D. A. Fishman, "Early detection of ovarian cancer with conventional and contrast-enhanced transvaginal sonography: recent advances and potential improvements," Journal of Oncology, vol. 2012, Article ID 302858, 11 pages, 2012. 